\title{
Bounding the Mim-Width of Hereditary Graph Classes
}

\author{
Nick Brettell \\ School of Mathematics and Statistics, Victoria University of Wellington, New Zealand \\ nick.brettell@vuw.ac.nz
}

Jake Horsfield

School of Computing, University of Leeds, UK

sc15jh@leeds.ac.uk

Andrea Munaro

School of Mathematics and Physics, Queen's University Belfast, UK

a.munaro@qub.ac.uk

Giacomo Paesani

Department of Computer Science, Durham University, UK

giacomo.paesani@durham.ac.uk

\section{Daniël Paulusma}

Department of Computer Science, Durham University, UK

daniel.paulusma@durham.ac.uk

\begin{abstract}
A large number of NP-hard graph problems are solvable in XP time when parameterized by some width parameter. Hence, when solving problems on special graph classes, it is helpful to know if the graph class under consideration has bounded width. In this paper we consider mim-width, a particularly general width parameter that has a number of algorithmic applications whenever a decomposition is "quickly computable" for the graph class under consideration.

We start by extending the toolkit for proving (un)boundedness of mim-width of graph classes. By combining our new techniques with known ones we then initiate a systematic study into bounding mim-width from the perspective of hereditary graph classes, and make a comparison with clique-width, a more restrictive width parameter that has been well studied.

We prove that for a given graph $H$, the class of $H$-free graphs has bounded mim-width if and only if it has bounded clique-width. We show that the same is not true for $\left(H_{1}, H_{2}\right)$-free graphs. We identify several general classes of $\left(H_{1}, H_{2}\right)$-free graphs having unbounded clique-width, but bounded mim-width, illustrating the power of mim-width. Moreover, we show that a branch decomposition of constant mim-width can be found in polynomial time, for these classes. Hence, as mentioned, these results have algorithmic implications: when the input is restricted to such a class of $\left(H_{1}, H_{2}\right)$-free graphs, many problems become polynomial-time solvable, including classical problems such as $k$-Colouring and IndEPENDENT SET, domination-type problems known as LC-VSVP problems, and distance versions of LC-VSVP problems, to name just a few. We also prove a number of new results showing that, for certain $H_{1}$ and $H_{2}$, the class of $\left(H_{1}, H_{2}\right)$-free graphs has unbounded mim-width.

Boundedness of clique-width implies boundedness of mim-width. By combining our results, which give both new bounded and unbounded cases for mim-width, with the known bounded cases for clique-width, we present summary theorems of the current state of the art for the boundedness of mim-width for $\left(H_{1}, H_{2}\right)$-free graphs. In particular, we classify the mim-width of $\left(H_{1}, H_{2}\right)$-free graphs for all pairs $\left(H_{1}, H_{2}\right)$ with $\left|V\left(H_{1}\right)\right|+\left|V\left(H_{2}\right)\right| \leq 8$. When $H_{1}$ and $H_{2}$ are connected graphs, we classify all pairs $\left(H_{1}, H_{2}\right)$ except for one remaining infinite family and a few isolated cases.
\end{abstract}

2012 ACM Subject Classification Theory of computation $\rightarrow$ Graph algorithms analysis

Keywords and phrases Width parameter, mim-width, clique-width, hereditary graph class

Digital Object Identifier 10.4230/LIPIcs.IPEC.2020.6

Funding The research in this paper received support from the Leverhulme Trust (RPG-2016-258).

cc (i) $\odot$ Nick Brettell, Jake Horsfield, Andrea Munaro, Giacomo Paesani, and Daniël Paulusma;

cc) licensed under Creative Commons License CC-BY

15th International Symposium on Parameterized and Exact Computation (IPEC 2020).

Editors: Yixin Cao and Marcin Pilipczuk; Article No. 6; pp. 6:1-6:18

Leibniz International Proceedings in Informatics

LI I I C S Schloss Dagstuhl - Leibniz-Zentrum für Informatik, Dagstuhl Publishing, Germany 


\section{Introduction}

Many computationally hard graph problems can be solved efficiently after placing appropriate restrictions on the input graph. Instead of trying to solve individual problems in an ad hoc way, one may aim to find the underlying reasons why some sets of problems behave better on certain graph classes than other sets of problems. The ultimate goal in this type of research is to obtain complexity dichotomies for large families of graph problems. Such dichotomies tell us for which graph classes a certain problem or set of problems can or cannot be solved efficiently (under standard complexity assumptions).

One reason that might explain the jump from computational hardness to tractability after restricting the input to some graph class $\mathcal{G}$ is that $\mathcal{G}$ has bounded "width", that is, every graph in $\mathcal{G}$ has width at most $c$ for some constant $c$. One can define the notion of "width" in many different ways (see the surveys [30, 31, 37, 47]). As such, the various width parameters differ in strength. To explain this, we say that a width parameter $p$ dominates a width parameter $q$ if there is a function $f$ such that $p(G) \leq f(q(G))$ for all graphs $G$. If $p$ dominates $q$ but $q$ does not dominate $p$, then $p$ is said to be more powerful than $q$. If both $p$ and $q$ dominate each other, then $p$ and $q$ are equivalent. For instance, the width parameters boolean-width, clique-width, module-width, NLC-width and rank-width are all equivalent $[15,36,42,44]$, but more powerful than the equivalent parameters branch-width and treewidth $[19,45,47]$.

In this paper we focus on an even more powerful width parameter called mim-width (maximum induced matching width). Vatshelle [47] introduced mim-width, which we define in Section 3, and proved that mim-width is more powerful than boolean-width, and consequently, clique-width, module-width, NLC-width and rank-width.

\subsection{Algorithmic Implications}

One trade-off of a more powerful width parameter is the difficulty in obtaining a branch decomposition of bounded width. In general, computing mim-width is NP-hard; deciding if the mim-width is at most $k$ is $\mathrm{W}[1]$-hard when parameterized by $k$; and there is no polynomial-time algorithm for approximating the mim-width of a graph to within a constant factor of the optimal, unless NP = ZPP [46]. Hence, in contrast to algorithms for graphs of bounded treewidth or clique-width, algorithms for graphs of bounded mim-width require a branch decomposition of constant mim-width as part of the input. On the other hand, there are many interesting graph classes for which mim-width is bounded and quickly computable, that is, the class admits a polynomial-time algorithm for obtaining a branch decomposition of constant mim-width. We give examples of such graph classes known in the literature in Section 1.2 before discussing the new graph classes we found in Section 1.4. Below we briefly discuss known algorithms for problems on graphs of bounded mim-width.

Belmonte and Vatshelle [1] and Bui-Xuan, Telle and Vatshelle [16] proved that a large set of problems, known as Locally Checkable Vertex Subset and Vertex Partitioning (LCVSVP) problems [43], can be solved in polynomial time for graph classes where mim-width is bounded and quickly computable. Well-known examples of such problems include (TOTAL) Dominating SEt, IndePEndent SET and $k$-Colouring for every fixed positive integer $k$. Later, Fomin, Golovach and Raymond [27] proved that the XP algorithms for INDEPENDENT SET and Dominating SET are in a sense best possible, showing that these two problems are W[1]-hard when parameterized by mim-width.

On the positive side, XP algorithms parameterized by mim-width are now also known for problems outside the LC-VSVP framework. In particular, Jaffke, Kwon, Strømme and Telle [33] proved that the distance versions of LC-VSVP problems can be solved in polynomial 
time for graph classes where mim-width is bounded and quickly computable. Jaffke, Kwon and Telle [34, 35] proved similar results for Longest Induced PATH, Induced Disjoint Paths, $H$-Induced Topological Minor and Feedback Vertex Set. The latter result has recently been generalized to Subset Feedback Vertex Set and Node Multiway Cut, by Bergougnoux, Papadopoulos and Telle [3].

Bergougnoux and Kanté [2] gave a meta-algorithm for problems with a global constraint, providing unifying XP algorithms in mim-width for several of the aforementioned problems, as well as Connected Dominating Set, Node Weighted Steiner Tree, and Maximum Induced Tree. Galby, Munaro and Ries [29] proved that Semitotal Dominating Set is polynomial-time solvable for graph classes where mim-width is bounded and quickly computable.

\subsection{Mim-width of Special Graph Classes}

Belmonte and Vatshelle [1] proved that the mim-width of the following graph classes is bounded and quickly computable: permutation graphs, convex graphs and their complements, interval graphs and their complements, circular $k$-trapezoid graphs, circular permutation graphs, Dilworth- $k$ graphs, $k$-polygon graphs, circular-arc graphs and complements of $d$ degenerate graphs.

Some of the results of [1] have been extended. Let $K_{r} \boxminus K_{r}$ be the graph obtained from $2 K_{r}$ by adding a perfect matching, and let $K_{r} \boxminus r P_{1}$ be the graph obtained from $K_{r} \boxminus K_{r}$ by removing all the edges in one of the complete graphs (see Section 2 for undefined notation). Kang et al. [38] showed that for any integer $r \geq 2$, there is a polynomial-time algorithm for computing a branch decomposition of mim-width at most $r-1$ when the input is restricted to $\left(K_{r} \boxminus r P_{1}\right)$-free chordal graphs, which generalize interval graphs, or $\left(K_{r} \boxminus K_{r}\right)$-free co-comparability graphs, which generalize permutation graphs. Hence, in particular, all these classes have bounded mim-width.

In addition to the above results, Kang et al. [38] proved that chordal graphs, circle graphs and co-comparability graphs have unbounded mim-width; Mengel [41] also proved, independently, that the latter two classes have unbounded mim-width. Vatshelle [47] and Brault-Baron et al. [11] showed the same for grids and chordal bipartite graphs, respectively, whereas Mengel [41] proved that strongly chordal split graphs have unbounded mim-width.

Brettell et al. [12] showed that the mim-width of $\left(K_{r}, s P_{1}+P_{5}\right)$-free graphs is bounded and quickly computable for every $r \geq 1$ and $s \geq 0$. In particular, this yielded an alternative proof for showing that LIST $k$-COLOURING is polynomially solvable for $\left(s P_{1}+P_{5}\right)$-free graphs for all $k \geq 1$ and $s \geq 0$ [20]. Let $K_{1, s}^{1}$ be the graph obtained from the $(s+1)$-vertex star $K_{1, s}$ after subdividing each edge once; note that $s P_{1}+P_{5}$ is an induced subgraph of $K_{1, s+2}^{1}$. In [13], the result of [12] on the mim-width of $\left(K_{r}, s P_{1}+P_{5}\right)$-free graphs was generalized to $\left(K_{r}, K_{1, s}^{1}, P_{t}\right)$-free graphs. As a consequence, for all $k \geq 3, s \geq 1$ and $t \geq 1$, LiST $k$-Colouring is polynomial-time solvable even for $\left(K_{1, s}^{1}, P_{t}\right)$-free graphs; previously this was shown for $k=3$ by Chudnovsky et al. [18].

Brettell et al. [14] considered the following generalisation of convex graphs. A bipartite graph $G=(A, B, E)$ is $\mathcal{H}$-convex, for some family of graphs $\mathcal{H}$, if there exists a graph $H \in \mathcal{H}$ with $V(H)=A$ such that the set of neighbours in $A$ of each $b \in B$ induces a connected subgraph of $H$ (when $\mathcal{H}$ is the set of paths, we obtain exactly convex graphs). They showed that the class of $\mathcal{H}$-convex graphs has bounded and quickly computable mim-width if $\mathcal{H}$ is the set of cycles, or $\mathcal{H}$ is the set of trees with bounded maximum degree and bounded number of vertices of degree at least 3 . 


\subsection{Our Focus}

We continue the study on boundedness of mim-width and aim to identify more graph classes of bounded or unbounded mim-width. Our motivation is both algorithmic and structural. As discussed above, there are clear algorithmic benefits if a graph class has bounded mim-width. From a structural point of view, we aim to initiate a systematic study of the boundedness of mim-width, comparable to a similar, long-standing study of the boundedness of clique-width (see [23] for a survey).

The framework of hereditary graph classes is highly suitable for such a study. A graph class $\mathcal{G}$ is hereditary if it is closed under vertex deletion. A class $\mathcal{G}$ is hereditary if and only if there exists a (unique) set of graphs $\mathcal{F}$ of (minimal) forbidden induced subgraphs for $\mathcal{G}$. That is, a graph $G$ belongs to $\mathcal{G}$ if and only if $G$ does not contain any graph from $\mathcal{F}$ as an induced subgraph. We also say that $G$ is $\mathcal{F}$-free. Note that $\mathcal{F}$ may have infinite size. For example, if $\mathcal{G}$ is the class of bipartite graphs, then $\mathcal{F}$ is the set of all odd cycles.

As a natural starting point we consider the case where $|\mathcal{F}|=1$, say $\mathcal{F}=\{H\}$. It is not difficult to verify that a class of $H$-free graphs has bounded mim-width if and only if it has bounded clique-width if and only if $H$ is an induced subgraph of the 4-vertex path $P_{4}$; see Section 3 for details. On the other hand, there exist hereditary graph classes, such as interval graphs and permutation graphs, that have bounded mim-width, even mim-width 1 [47], but unbounded clique-width [32]. However, these graph classes have an infinite set of forbidden induced subgraphs. Hence, questions we aim to address in this paper are: Does there exist a hereditary graph class characterized by a finite set $\mathcal{F}$ that has bounded mim-width but unbounded clique-width? Can we use the same techniques as when dealing with clique-width? In particular we will focus on the case where $|\mathcal{F}|=2$, say $\mathcal{F}=\left\{H_{1}, H_{2}\right\}$. Such classes are called bigenic.

\subsection{Our Results and Methodology}

In order to work with width parameters it is useful to have a set of graph operations that preserve boundedness or unboundedness of the width parameter. That is, if we apply such a width-preserving operation, or only apply it a constant number of times, the width of the graph does not change by too much. In this way one might be able to modify an arbitrary graph from a given "unknown" class $\mathcal{G}_{1}$ into a graph from a class $\mathcal{G}_{2}$ known to have bounded or unbounded width. This would then imply that $\mathcal{G}_{1}$ also has bounded or unbounded width, respectively. Two useful operations preserving clique-width are vertex deletion [40] and subgraph complementation [37]. The latter operation replaces every edge in some subgraph of the graph by a non-edge, and vice versa. As we will see in Section 6, subgraph complementation does not preserve boundedness or unboundedness of mim-width ${ }^{1}$.

To work around this limitation, we collect and generalize known mim-width preserving graph operations from the literature in Section 3 (some of these operations only show that the mim-width cannot decrease after applying them). In the same section we also state some known useful results on mim-width and prove that elementary graph classes, such as walls and net-walls, have unbounded mim-width. In Sections 4 and 5 we use the results from Section 3. In Section 4 we present new bigenic classes of bounded mim-width. These graph classes are all known to have unbounded clique-width. Hence, our results show that the dichotomy for boundedness of mim-width no longer coincides with the one for clique-width when $|\mathcal{F}|=2$ instead of $|\mathcal{F}|=1$. Moreover, for each of these classes, a branch decomposition

1 The situation is different for mim-width 1 ; Vatshelle $[47]$ showed that if $\operatorname{mimw}(G)=1$ then $\operatorname{mimw}(\bar{G})=1$. 


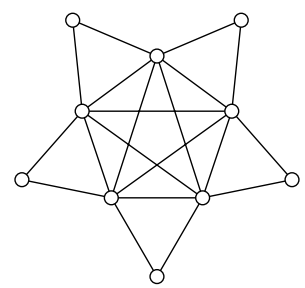

Figure 1 The graph $\operatorname{sun}_{5}$.

of constant mim-width is easily computable for any graph in the class. This immediately implies that there are polynomial-time algorithms for many problems when restricted to these classes, as described in Section 1.1. In Section 5 we present new bigenic classes of unbounded mim-width; these graph classes are known to have unbounded clique-width. In Section 6 we give a state-of-the-art summary of our new results combined with known results. The known results include the bigenic graph classes of bounded clique-width (as bounded clique-width implies bounded mim-width). In the same section we compare our results for the mim-width of bigenic graph classes with the ones for clique-width. We also state a number of open problems.

\section{Preliminaries}

We consider only finite graphs $G=(V, E)$ with no loops and no multiple edges. For a vertex $v \in V$, the neighbourhood $N(v)$ is the set of vertices adjacent to $v$ in $G$. The degree $d(v)$ of a vertex $v \in V$ is the size $|N(v)|$ of its neighbourhood. A graph is subcubic if every vertex has degree at most 3. For disjoint $S, T \subseteq V$, we say that $S$ is complete to $T$ if every vertex of $S$ is adjacent to every vertex of $T$, and $S$ is anticomplete to $T$ if there are no edges between $S$ and $T$. The distance from a vertex $u$ to a vertex $v$ in $G$ is the length of a shortest path between $u$ and $v$. A set $S \subseteq V$ induces the subgraph $G[S]=(S,\{u v: u, v \in S, u v \in E\})$. If $G^{\prime}$ is an induced subgraph of $G$ we write $G^{\prime} \subseteq_{i} G$. The complement of $G$ is the graph $\bar{G}$ with vertex set $V(G)$, such that $u v \in E(\bar{G})$ if and only if $u v \notin E(G)$.

Given a graph $G$ and a degree- $k$ vertex $v$ of $G$ with $N(v)=\left\{u_{1}, \ldots, u_{k}\right\}$, the clique implant on $v$ is the operation of deleting $v$, adding $k$ new vertices $v_{1}, \ldots, v_{k}$ forming a clique, and adding edges $v_{i} u_{i}$ for each $i \in\{1, \ldots, k\}$. The $k$-subdivision of an edge $u v$ in a graph replaces $u v$ by $k$ new vertices $w_{1}, \ldots, w_{k}$ with edges $u w_{1}, w_{k} v$ and $w_{i} w_{i+1}$ for each $i \in\{1, \ldots, k-1\}$, i.e. the edge is replaced by a path of length $k+1$. The disjoint union $G+H$ of graphs $G$ and $H$ has vertex set $V(G) \cup V(H)$ and edge set $E(G) \cup E(H)$. We denote the disjoint union of $k$ copies of $G$ by $k G$. For a graph $H$, a graph $G$ is $H$-free if $G$ has no induced subgraph isomorphic to $H$. For a set of graphs $\left\{H_{1}, \ldots, H_{k}\right\}$, a graph $G$ is $\left(H_{1}, \ldots, H_{k}\right)$-free if $G$ is $H_{i}$-free for every $i \in\{1, \ldots, k\}$.

An independent set of a graph is a set of pairwise non-adjacent vertices. A clique of a graph is a set of pairwise adjacent vertices. A matching of a graph is a set of pairwise non-adjacent edges. A matching $M$ of a graph $G$ is induced if there are no edges of $G$ between vertices incident to distinct edges of $M$.

The path, cycle and complete graph on $n$ vertices are denoted by $P_{n}, C_{n}$ and $K_{n}$, respectively. The graph $K_{3}$ is also called the triangle. A graph is $r$-partite, for $r \geq 2$, if its vertex set admits a partition into $r$ classes such that every edge has its endpoints in different classes. An $r$-partite graph in which every two vertices from different partition classes are adjacent is a complete r-partite graph and a 2-partite graph is also called bipartite. 
A graph is co-bipartite if it is the complement of a bipartite graph. A split graph is a graph $G$ that admits a split partition $(C, I)$, that is, $V(G)$ can be partitioned into a clique $C$ and an independent set $I$. Equivalently, a graph is split if and only if it is $\left(2 P_{2}, C_{4}, C_{5}\right)$-free. The subdivided claw $S_{h, i, j}$, for $1 \leq h \leq i \leq j$ is the tree with one vertex $x$ of degree 3 and exactly three leaves, which are of distance $h, i$ and $j$ from $x$, respectively. Note that $S_{1,1,1}=K_{1,3}$. For $t \geq 3$ sun $_{t}$ denotes the graph on $2 t$ vertices obtained from a complete graph on $t$ vertices $u_{1}, \ldots, u_{t}$ by adding $t$ vertices $v_{1}, \ldots, v_{t}$ such that $v_{i}$ is adjacent to $u_{i}$ and $u_{i+1}$ for each $i \in\{1, \ldots, t-1\}$ and $v_{t}$ is adjacent to $u_{1}$ and $u_{t}$. See Figure 1 for a picture of $\operatorname{sun}_{5}$.

\section{Mim-Width: Definition and Basic Results}

A branch decomposition for a graph $G$ is a pair $(T, \delta)$, where $T$ is a subcubic tree and $\delta$ is a bijection from $V(G)$ to the leaves of $T$. Each edge $e \in E(T)$ naturally partitions the leaves of $T$ into two classes, depending on which component they belong to when $e$ is removed. In this way, each edge $e \in E(T)$ corresponds to a partition $L_{e}$ and $\overline{L_{e}}$ of the set of leaves of $T$, depending on which component of $T-e$ the leaves of $T$ belong to. Consequently, each edge $e$ induces a partition $\left(A_{e}, \overline{A_{e}}\right)$ of $V(G)$, where $\delta\left(A_{e}\right)=L_{e}$ and $\delta\left(\overline{A_{e}}\right)=\overline{L_{e}}$. For two disjoint sets $X$ and $Y$, let $G[X, Y]$ denote the bipartite subgraph of $G$ induced by the edges with one endpoint in $X$ and the other in $Y$. For each edge $e \in E(T)$ and corresponding partition $\left(A_{e}, \overline{A_{e}}\right)$ of $V(G)$, we denote by $\operatorname{cutmim}_{G}\left(A_{e}, \overline{A_{e}}\right)$ the size of a maximum induced matching in $G\left[A_{e}, \overline{A_{e}}\right]$. The mim-width of the branch decomposition $(T, \delta)$ is the quantity $\operatorname{mimw}_{G}(T, \delta)=\max _{e \in E(T)} \operatorname{cutmim}_{G}\left(A_{e}, \overline{A_{e}}\right)$. The mim-width of the graph $G$, denoted $\operatorname{mimw}(G)$, is the minimum value of $\operatorname{mimw}_{G}(T, \delta)$ over all possible branch decompositions $(T, \delta)$ for $G$.

Mim-Width Preserving Operations. The following three lemmas, the first of which is due to Vatshelle, show that vertex deletion, edge subdivision and clique implantation do not change the mim-width of a graph by too much. We omit the proofs of the second and third lemma.

- Lemma 1 ([47]). Let $G$ be a graph and $v \in V(G)$. Then $\operatorname{mimw}(G)-1 \leq \operatorname{mimw}(G-v) \leq$ $\operatorname{mimw}(G)$.

- Lemma 2. Let $G$ be a graph and let $G^{\prime}$ be the graph obtained by 1-subdividing an edge of $G$. Then $\operatorname{mimw}(G) \leq \operatorname{mimw}\left(G^{\prime}\right) \leq \operatorname{mimw}(G)+1$.

- Lemma 3. Let $G$ be a graph and let $G^{\prime}$ be the graph obtained from $G$ by a clique implant on $v \in V(G)$. Then $\operatorname{mimw}(G) \leq \operatorname{mimw}\left(G^{\prime}\right) \leq \operatorname{mimw}(G)+d(v)$.

Mengel [41] showed that adding edges inside the partition classes of a bipartite graph does not decrease mim-width by much. This result can be generalized to $k$-partite graphs in the following way.

- Lemma 4. Let $G$ be a k-partite graph with partition classes $V_{1}, \ldots, V_{k}$, and let $G^{\prime}$ be a graph obtained from $G$ by adding edges where for each added edge, there exists some $i$ such that both endpoints are in $V_{i}$. Then $\operatorname{mimw}\left(G^{\prime}\right) \geq \frac{1}{k} \cdot \operatorname{mimw}(G)$.

Proof. Let $(T, \delta)$ be a branch decomposition for $G^{\prime}$. Since $G$ and $G^{\prime}$ have the same vertex set, $(T, \delta)$ is a branch decomposition for $G$ as well. It is enough to show that $\operatorname{mimw}_{G}(T, \delta) \leq$ $k \cdot \operatorname{mimw}_{G^{\prime}}(T, \delta)$. Therefore, let $e \in E(T)$ be such that $\operatorname{mimw}_{G}(T, \delta)=\operatorname{cutmim}_{G}\left(A_{e}, \overline{A_{e}}\right)$, and let $M$ be a maximum induced matching in $G\left[A_{e}, \overline{A_{e}}\right]$. For each $i$, consider the set 
$M_{i}=\left\{u v \in M: u \in A_{e} \cap V_{i}\right\}$. These $k$ sets partition $M$. Let $M^{\prime}$ be a partition class of size at least $|M| / k$. Clearly, $M^{\prime}$ is an induced matching in $G^{\prime}\left[A_{e}, \overline{A_{e}}\right]$ and so $k \cdot \operatorname{mimw}_{G^{\prime}}(T, \delta) \geq k \cdot\left|M^{\prime}\right| \geq|M|=\operatorname{mimw}_{G}(T, \delta)$.

The next lemma shows that to bound the mim-width of a class of graphs, we may restrict our attention to 2-connected graphs in the class. We omit the proof and note that this property is not specific to mim-width: it has also been observed, in [30], for rank-width, and this argument also applies for any appropriate width parameter defined using branch decompositions. A block is a maximal connected subgraph with no cut-vertex.

- Lemma 5. Let $G$ be a graph. Then $\operatorname{mimw}(G)=\max \{\operatorname{mimw}(H): H$ is a block of $G\}$. Moreover, given branch decompositions $\left(T_{H}, \delta_{H}\right)$ of each block $H$ of $G$, with $\operatorname{mimw}_{H}\left(T_{H}, \delta_{H}\right) \leq$ $k$, we can compute a branch decomposition of $G$ with mim-width at most $k$ in polynomial time.

The following lemma is due to Galby and Munaro, who used it to prove that Dominating SET admits a PTAS for a subclass of VPG graphs when the representation is given.

- Lemma 6 ([28]). Let $G$ be a graph and let $S \subseteq V$. Let $G^{\prime}=\left(V^{\prime}, E^{\prime}\right)$ denote the graph with $V^{\prime}=V$ and $E^{\prime}=E \cup\{u v: u, v \in S\}$. Then $\operatorname{mimw}\left(G^{\prime}\right) \leq \operatorname{mimw}(G)+1$.

The final structural lemma is used to prove that $\left(s P_{1}+P_{5}, K_{t}\right)$-free graphs have bounded mim-width for every $s \geq 0$ and $t \geq 1$. It shows how we can bound the mim-width of a graph in terms of the mim-width of the graphs induced by blocks of a partition of the vertex set and the mim-width between any two of the parts. We include it here as it might be useful for bounding the mim-width of other graph classes.

- Lemma 7 ([12]). Let $G$ be a graph and $\left(X_{1}, \ldots, X_{p}\right)$ be a partition of $V(G)$ such that $\operatorname{cutmim}_{G}\left(X_{i}, X_{j}\right) \leq c$ for all distinct $i, j \in\{1, \ldots, p\}$, and $p \geq 2$. Then

$$
\operatorname{mimw}(G) \leq \max \left\{c\left\lfloor\left(\frac{p}{2}\right)^{2}\right\rfloor, \max _{i \in\{1, \ldots, p\}}\left\{\operatorname{mimw}\left(G\left[X_{i}\right]\right)\right\}+c(p-1)\right\} .
$$

Moreover, if $\left(T_{i}, \delta_{i}\right)$ is a branch decomposition of $G\left[X_{i}\right]$ for each $i$, then we can construct, in $O(1)$ time, a branch decomposition $(T, \delta)$ of $G$ with

$$
\operatorname{mimw}_{G}(T, \delta) \leq \max \left\{c\left\lfloor\left(\frac{p}{2}\right)^{2}\right\rfloor, \max _{i \in\{1, \ldots, p\}}\left\{\operatorname{mimw}_{G}\left(T_{i}, \delta_{i}\right)\right\}+c(p-1)\right\} .
$$

Mim-width of Some Basic Classes. Recall that Vatshelle [47] showed that the class of grids has unbounded mim-width. We next prove that the same holds for the class of walls, which we define momentarily. Thus, we obtain a class of graphs with maximum degree 3 having unbounded mim-width, and we will use this result in order to prove Lemma 11. Note that it also gives us a dichotomy, as graphs with maximum degree 2 have bounded clique-width and hence bounded mim-width.

A wall of height $h$ and width $r$ (an $(h \times r)$-wall for short) is the graph obtained from the grid of height $h$ and width $2 r$ as follows. Let $C_{1}, \ldots, C_{2 r}$ be the set of vertices in each of the $2 r$ columns of the grid, in their natural left-to-right order. For each column $C_{j}$, let $e_{1}^{j}, e_{2}^{j}, \ldots, e_{h-1}^{j}$ be the edges between two vertices of $C_{j}$, in their natural top-to-bottom order. If $j$ is odd, we delete all edges $e_{i}^{j}$ with $i$ even. If $j$ is even, we delete all edges $e_{i}^{j}$ with $i$ odd. We then remove all vertices of the resulting graph whose degree is 1 . This final graph is an elementary $(h \times r)$-wall and any subdivision of the elementary $(h \times r)$-wall is an $(h \times r)$-wall. For an example, see Figure 2. 


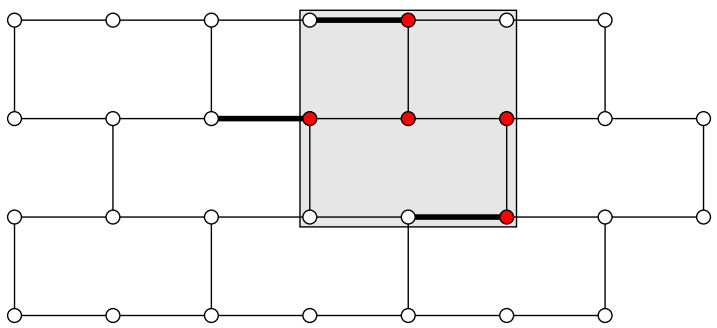

Figure 2 An elementary $(4 \times 4)$-wall. We illustrate an example of the case where $h \geq \sqrt[4]{n(W) / 3}$ and $r<2 n$ in the proof of Theorem 8: $Q$ consists of the red vertices, $B$ is the the grey box, and the thick edges are a matching in $W\left[A_{e}, \overline{A_{e}}\right]$.

- Theorem 8. Let $W$ be an elementary $(n \times n)$-wall with $n \geq 7$. Then $\operatorname{mimw}(W) \geq \frac{\sqrt{n}}{50}$. In particular, the class of walls has unbounded mim-width.

Proof. We let $n(W)=|V(W)|=2 n^{2}-2$. Consider now a branch decomposition $(T, \delta)$ for $W$. There exists $e \in E(T)$ such that both partition classes $A_{e}$ and $\overline{A_{e}}$ of $V(W)$ contain at least $n(W) / 3$ vertices [38, Lemma 2.3]. Kanj et al. [39, Lemma 4.10] showed that if $G$ is a graph such that each of its subgraphs has average degree at most $d$, then any matching $M$ in $G$ contains an induced matching in $G$ of size at least $|M| /(2 d-1)$. Since $W$ is subcubic, it is sufficient to show that $W\left[A_{e}, \overline{A_{e}}\right]$ has a matching of size $\sqrt{n} / 10$. We distinguish two cases, according to whether or not one of $W\left[A_{e}\right]$ and $W\left[\overline{A_{e}}\right]$ has a component of size at least $\sqrt{n(W) / 3}$.

Suppose first that $W\left[A_{e}\right]$ has a component $Q$ of size at least $\sqrt{n(W) / 3}$. The component $Q$ is contained in a rectangle of the underlying $n \times 2 n$ grid. Consider the smallest such rectangle $B$, i.e., the rectangle whose horizontal sides contain the uppermost and lowermost vertex in $Q$ and whose vertical sides contain the leftmost and rightmost vertex in $Q$. Let $h$ and $r$ be the height and width of $B$, respectively. Since $|V(Q)| \geq \sqrt{n(W) / 3}$, one of $h$ and $r$ is at least $\sqrt[4]{n(W) / 3}$.

Suppose first that $h \geq \sqrt[4]{n(W) / 3}$. If $r<2 n$, say without loss of generality $B$ does not intersect column $C_{1}$, we do the following. For each row of $B$, consider the leftmost vertex of $Q$ in that row (since $Q$ is connected, each row contains at least one vertex of $Q$ ). Clearly, the left neighbours of each such vertex belongs to $\overline{A_{e}}$, and so we have a matching in $W\left[A_{e}, \overline{A_{e}}\right]$ of size $h-2 \geq \sqrt[4]{n(W) / 3}-2$, which is at least $\sqrt{n} / 10$ when $n \geq 7$. If $r=2 n$, we distinguish two cases according to whether $h=n$ or not. In the first case (i.e., $r=2 n$ and $h=n$ ) we argue as follows. Since $Q$ is connected, each row of $B$ contains a vertex of $Q \subseteq A_{e}$. Moreover, there are at most $2 n / 3$ rows of $B$ with all vertices contained in $A_{e}$, for otherwise $\left|A_{e}\right|>(2 n / 3) \cdot 2 n \geq 2 n(W) / 3$. So there are at least $n / 3$ rows of $B$ containing a vertex of $A_{e}$ and a vertex of $\overline{A_{e}}$. We can therefore find a matching in $W\left[A_{e}, \overline{A_{e}}\right]$ of size at least $n / 3$. In the second case (i.e., $r=2 n$ and $h<n$ ), we proceed as follows. We assume, without loss of generality, that $B$ does not intersect the uppermost row of the grid. We partition the columns of $B$ into disjoint layers containing two consecutive columns each. For each layer, we consider its left column and the uppermost vertex $v \in A_{e}$ therein (since $Q$ is connected, such a vertex exists). Let $v_{1}$ be the vertex on the grid above $v$, let $v_{2}$ be the vertex to the right of $v$ and let $v_{3}$ be the vertex above $v_{2}$. By construction, $v_{1} \in \overline{A_{e}}$ and if $v v_{1} \in E(W)$, we select this edge. Otherwise, $v v_{1} \notin E(W)$ and so $v_{2} v_{3} \in E(W)$ and we have a path $v v_{2} v_{3} v_{1}$ in $W$ with $v \in A_{e}$ and $v_{1} \in \overline{A_{e}}$. We then select an edge of this path which belongs to $W\left[A_{e}, \overline{A_{e}}\right]$. Proceeding similarly for each layer, we obtain a matching in $W\left[A_{e}, \overline{A_{e}}\right]$ of size at least $r / 2=n$. Suppose finally that $h<\sqrt[4]{n(W) / 3}$. We have that $r \geq \sqrt[4]{n(W) / 3}$ and we proceed exactly as in the case $r=2 n$ and $h<n$ to obtain a matching in $W\left[A_{e}, \overline{A_{e}}\right]$ of size 
at least $r / 2 \geq \sqrt[4]{n(W) / 3} / 2$.

It remains to consider the situation in which all components of $W\left[A_{e}\right]$ and $W\left[\overline{A_{e}}\right]$ have size less than $\sqrt{n(W) / 3}$. In particular, since $W\left[A_{e}\right]$ has more than $n(W) / 3$ vertices, it has more than $\sqrt{n(W) / 3}$ components. Let $Q_{1}, \ldots, Q_{k}$ be these components. For each $i \in\{1, \ldots, k\}$, there exists a vertex $u_{i} \in Q_{i}$ with a neighbour $v_{i} \in \overline{A_{e}}$, as $W$ is connected. Let $H$ be the subgraph of $W\left[A_{e}, \overline{A_{e}}\right]$ induced by $\left\{u_{1}, \ldots, u_{k}\right\} \cup\left\{v_{1}, \ldots, v_{k}\right\}$ (notice that we might have $v_{i}=v_{j}$ for some $\left.i \neq j\right)$. Let $H_{1}, \ldots, H_{\ell}$ be the components of $H$ and let $n_{i}=\left|V\left(H_{i}\right)\right|$, for each $i \in\{1, \ldots, \ell\}$. By construction, $n_{i} \geq 2$, for each $i$. Moreover, since $H_{i}$ is a connected subcubic graph, it has a matching of size at least $\left(n_{i}-1\right) / 3 \geq n_{i} / 6$ [4]. But then $H$ has a matching of size

$$
\sum_{i=1}^{\ell} \frac{n_{i}}{6}=\frac{|V(H)|}{6} \geq \frac{k}{6} \geq \frac{1}{6} \cdot \sqrt{\frac{n(W)}{3}} .
$$

As in all cases we find a matching in $W\left[A_{e}, \overline{A_{e}}\right]$ of size at least $\frac{\sqrt{n}}{10}$, this concludes the proof.

- Corollary 9. For an integer $\Delta$, let $\mathcal{G}_{\Delta}$ be the class of graphs of maximum degree at most $\Delta$. Then the mim-width of $\mathcal{G}_{\Delta}$ is bounded if and only if $\Delta \leq 2$.

A net-wall is a graph that can be obtained from a wall $G$ by performing a clique implant on each vertex of $G$ having degree three. An example of part of a net-wall is given in Figure 4 .

The following lemma is a straightforward consequence of Theorem 8 and Lemma 3.

- Lemma 10. The class of net-walls has unbounded mim-width.

Mengel [41] showed that strongly chordal split graphs, or equivalently $\left(\operatorname{sun}_{3}, \operatorname{sun}_{4}, \ldots\right)$-free split graphs, have unbounded mim-width. We find two more subclasses of split graphs with unbounded mim-width by using Lemmas 2 and 4 .

- Lemma 11. Let $\mathcal{G}$ be the class of split graphs, or equivalently $\left(C_{4}, C_{5}, 2 P_{2}\right)$-free graphs, where one of the following properties is satisfied by every $G \in \mathcal{G}$ :

(i) $G$ has a split partition $(C, I)$ where each vertex in $I$ has degree 2 and each vertex in $C$ has at most three neighbours in $I$,

(ii) $G$ has a split partition $(C, I)$ where each vertex in $I$ has degree at most 3 , and each vertex in $C$ has two neighbours in $I$, or

(iii) $G$ is sun $_{t}$-free $t \geq 3$.

Then $\mathcal{G}$ has unbounded mim-width.

Proof. Statement (iii) is due to Mengel [41]. To prove (i) and (ii), let $G$ be a wall, and let $G^{\prime}$ be the graph obtained by 1-subdividing each edge of $G$. Partition $V\left(G^{\prime}\right)$ into $(A, B)$, where $B$ consists of the vertices of degree two introduced by the 1-subdivisions. Observe that $G^{\prime}$ is bipartite, with vertex bipartition $(A, B)$. Let $G^{\prime \prime}$ be the graph obtained by making one of $A$ or $B$ a clique. By Lemmas 2 and $4, \operatorname{mimw}\left(G^{\prime \prime}\right) \geq \operatorname{mimw}(G) / 2$. The result now follows from Theorem 8 .

A graph is chordal bipartite if it is bipartite and every induced cycle has four vertices. Brault-Baron et al. [11] showed that the class of chordal bipartite graphs has unbounded mim-width. Combining their result with Lemma 4, after adding all edges in a colour class, yields the following:

- Lemma 12. The class of co-bipartite graphs, or equivalently $\left(3 P_{1}, C_{5}, \overline{C_{7}}, \overline{C_{9}}, \ldots\right)$-free graphs, has unbounded mim-width. 
As the last result in this section we consider hereditary classes defined by one forbidden induced subgraph. It is folklore that the class of $H$-free graphs has bounded clique-width if and only if $H \subseteq_{i} P_{4}$ (see [26] for a proof). It turns out that the same dichotomy holds for mim-width.

- Theorem 13. The class of $H$-free graphs has bounded mim-width if and only if $H \subseteq_{i} P_{4}$.

Proof. If $H \subseteq_{i} P_{4}$, then $H$-free graphs form a subclass of $P_{4}$-free graphs. Every $P_{4}$-free graph has clique-width at most 2 [19] and so mim-width at most 2 [47]. Suppose now that $H$ is a graph such that the class of $H$-free graphs has bounded mim-width. Recall that chordal bipartite graphs have unbounded mim-width [11] (see also Section 5). Hence, $H$ is $C_{3}$-free. As co-bipartite graphs, and thus $3 P_{1}$-free graphs, and split graphs, or equivalently, $\left(C_{4}, C_{5}, 2 P_{2}\right)$-free graphs, have unbounded mim-width by Lemmas 11 and 12, this means that $H$ is a $\left(3 P_{1}, 2 P_{2}\right)$-free forest. It follows that $H \subseteq_{i} P_{4}$.

\section{$4 \quad$ New Bounded Cases}

In this section, we present three general classes, and two further specific classes, of $\left(H_{1}, H_{2}\right)$ free graphs having bounded mim-width, but unbounded clique-width. First, we present the three infinite families of classes of $\left(H_{1}, H_{2}\right)$-free graphs. We show that for a class in one of these three families, there exists a constant $k$ such that for every graph $G$ in the class, and every $X \subseteq V(G)$, we have that $\operatorname{cutmim}_{G}(X, \bar{X}) \leq k$. This implies that every branch decomposition of $G$ has mim-width at most $k$. Thus, for a graph in one of these classes, a branch decomposition of constant mim-width is quickly computable: any branch decomposition will suffice. Finally, we present two more classes of $\left(H_{1}, H_{2}\right)$-free graphs having bounded mim-width, which do not have this property, but for which we prove that a branch decomposition of constant width can be computed in polynomial-time.

We make use of Ramsey theory. By Ramsey's Theorem, for all positive integers $a$ and $b$, there exists an integer $R(a, b)$ such that if $G$ is a graph on at least $R(a, b)$ vertices, then $G$ has either a clique of size $a$, or an independent set of size $b$.

Recall that $K_{r} \boxminus K_{r}$ is the graph obtained from $2 K_{r}$ by adding a perfect matching and that $K_{r} \boxminus r P_{1}$ is the graph obtained from $K_{r} \boxminus K_{r}$ by removing all the edges in one of the complete graphs. We let $K_{r} \boxminus P_{1}$ denote the graph obtained from $K_{r}$ by adding a single vertex, attached to $K_{r}$ by a single pendant edge. We also denote $\overline{C_{4}+P_{1}}$ as bowtie. Examples of these graphs are given in Figure 3.
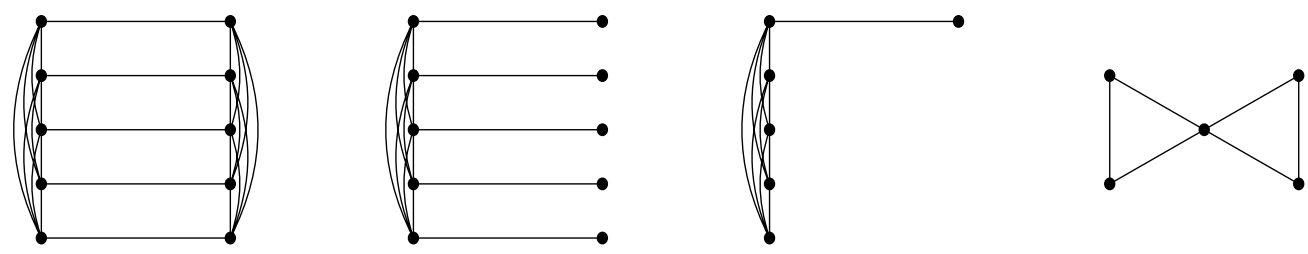

Figure 3 The graphs $K_{5} \boxminus K_{5}, K_{5} \boxminus 5 P_{1}, K_{5} \boxminus P_{1}$, and bowtie $=\overline{C_{4}+P_{1}}$.

- Theorem 14. Let $G$ be a $\left(K_{r} \boxminus r P_{1}, 2 P_{2}\right)$-free graph for $r \geq 3$. Then cutmim $_{G}(X, \bar{X})<$ $\max \{6, r\}$ for every $X \subseteq V(G)$. In particular, $\operatorname{mimw}(G)<\max \{6, r\}$.

Proof. Let $k=\max \{6, r\}$ and let $(T, \delta)$ be a branch decomposition of $G$. Towards a contradiction, suppose that there exists $X \subseteq V(G)$ such that $G[X, \bar{X}]$ has an induced matching of size at least $k$. Let $X^{\prime}=\left\{x_{1}, x_{2}, \ldots, x_{k}\right\} \subseteq X$ and $Y^{\prime}=\left\{y_{1}, y_{2}, \ldots, y_{k}\right\} \subseteq \bar{X}$ such that $x_{i} y_{i}$ is an edge of the induced matching for each $i \in\{1,2, \ldots, k\}$. 
First, observe that for any distinct $i, j \in\{1,2, \ldots, k\}$, either $x_{i} x_{j}$ or $y_{i} y_{j}$ is an edge, otherwise $G\left[\left\{x_{i}, x_{j}, y_{i}, y_{j}\right\}\right] \cong 2 P_{2}$. We claim that $X^{\prime}$ or $Y^{\prime}$ contains a clique of size 3 . Since $\left|X^{\prime}\right|=k \geq 6=R(3,3)$, the set $X^{\prime}$ contains either a clique on 3 vertices, or an independent set on 3 vertices. So we may assume that $X^{\prime}$ contains an independent set on 3 vertices, $\left\{x_{i}, x_{j}, x_{\ell}\right\}$ say. Then $\left\{y_{i}, y_{j}, y_{\ell}\right\}$ is a clique of size 3 contained in $Y^{\prime}$, proving the claim.

Without loss of generality, we may now assume that $X^{\prime}$ contains a clique of size 3 . Suppose $X^{\prime}$ is not a clique. Then there exist distinct $i, j \in\{1,2, \ldots, k\}$ such that $x_{i}$ is not adjacent to $x_{j}$. Now $y_{i} y_{j}$ is an edge, since $G$ is $2 P_{2}$-free. Let $X^{\prime \prime}$ be a maximum-sized clique contained in $X^{\prime}$, so $\left|X^{\prime \prime}\right| \geq 3$. Note that $\left\{x_{i}, x_{j}\right\} \nsubseteq X^{\prime \prime}$, since $X^{\prime \prime}$ is a clique, so we may assume that $x_{j} \notin X^{\prime \prime}$. As any pair in $X^{\prime \prime} \backslash\left\{x_{i}\right\}$ induces an edge that is anticomplete to the edge $y_{i} y_{j}$, we see that $G$ contains an induced $2 P_{2}$, a contradiction. We deduce that $X^{\prime}$ is a clique of size $k$. Now, since $G$ is $\left(K_{r} \boxminus r P_{1}\right)$-free, there exist distinct $i, j \in\{1,2, \ldots, k\}$ such that $y_{i} y_{j}$ is an edge. Note that since $k \geq 6$, there exist distinct $s, t \in\{1,2, \ldots, k\} \backslash\{i, j\}$. But now $x_{s} x_{t}$ is anticomplete to $y_{i} y_{j}$, contradicting that $G$ is $2 P_{2}$-free.

The class of $\left(K_{r} \boxminus r P_{1}, 2 P_{2}\right)$-free graphs for $r \in\{1,2\}$ is a subclass of $P_{4}$-free graphs, and thus has bounded clique-width and mim-width. However, for $r \geq 3$, the class of $\left(K_{r} \boxminus r P_{1}, 2 P_{2}\right)$ free graphs has unbounded clique-width [23, Theorem 4.18], whereas Theorem 14 shows it has bounded mim-width. In particular, (net, $2 P_{2}$ )-free graphs and (bull, $2 P_{2}$ )-free graphs have bounded mim-width but unbounded clique-width.

In our next two results, we present two other new classes of bounded mim-width. We omit the proof of the second result.

- Theorem 15. Let $G$ be a $\left(K_{r} \boxminus P_{1}, t P_{2}\right)$-free graph for $r \geq 1$ and $t \geq 1$. Then $\operatorname{cutmim}_{G}(X, \bar{X})<R(r, R(r, t))$ for every $X \subseteq V(G)$. In particular, $\operatorname{mimw}(G)<R(r, R(r, t))$.

Proof. Let $k=R(r, R(r, t))$ and let $(T, \delta)$ be a branch decomposition of $G$. Towards a contradiction, suppose that there exists $X \subseteq V(G)$ such that $G[X, \bar{X}]$ has an induced matching of size at least $k$. Let $X^{\prime}=\left\{x_{1}, x_{2}, \ldots, x_{k}\right\} \subseteq X$ and $Y^{\prime}=\left\{y_{1}, y_{2}, \ldots, y_{k}\right\} \subseteq \bar{X}$ such that $x_{i} y_{i}$ is an edge of the induced matching for each $i \in\{1,2, \ldots, k\}$.

Since $\left|X^{\prime}\right|=k=R(r, R(r, t))$, the set $X^{\prime}$ contains either a clique of size $r$, or an independent set of size $R(r, t)$. Suppose there is some $J \subseteq\{1,2, \ldots, k\}$ such that $X_{J}=\left\{x_{i}\right.$ : $i \in J\}$ is a clique of size $r$. Then, for an arbitrarily chosen $j \in J$, the vertices $X_{J} \cup\left\{y_{j}\right\}$ induce a $K_{r} \boxminus P_{1}$, a contradiction. So $X^{\prime}$ contains an independent set of size $R(r, t)$. Let $I \subseteq\{1,2, \ldots, k\}$ such that $X_{I}=\left\{x_{i}: i \in I\right\}$ is an independent set of size $R(r, t)$, and consider the set $Y_{I}=\left\{y_{i}: i \in I\right\}$. Since $\left|Y_{I}\right|=R(r, t)$, the set $Y_{I}$ either contains a clique of size $r$, or an independent set of size $t$. In the former case, $G$ contains an induced $K_{r} \boxminus P_{1}$, while in the latter case, $G$ contains an induced $t P_{2}$, a contradiction.

Theorem 16. Let $G$ be a $\left(K_{r} \boxminus K_{r}, s P_{1}+P_{2}\right)$-free graph for $r \geq 1$ and $s \geq 0$. Then cutmim $_{G}(X, \bar{X})<R(R(r, s+1), s+1)$ for every $X \subseteq V(G)$. In particular, $\operatorname{mimw}(G)<$ $R(R(r, s+1), s+1)$.

Note that $\left(K_{r} \boxminus P_{1}, t P_{2}\right)$-free graphs have unbounded clique-width if and only if $r \geq 3, t \geq 3$, or $r \geq 4, t \geq 2$ [23, Theorem 4.18]. Note also that $\left(K_{r} \boxminus K_{r}, s P_{1}+P_{2}\right)$-free graphs have unbounded clique-width if and only if $r=2, s \geq 3$, or $r \geq 3, s \geq 2$ [23, Theorem 4.18].

Our final results of the section resolve the remaining cases where $\left|V\left(H_{1}\right)\right|+\left|V\left(H_{2}\right)\right| \leq 8$ (we omit their proofs). For these results, we employ the following approach. Suppose we wish to show that the class of $\left(H_{1}^{\prime}, H_{2}^{\prime}\right)$-free graphs is bounded, where $H_{1}^{\prime} \subseteq_{i} H_{1}$ for one of the pairs $\left(H_{1}, H_{2}\right)$ appearing in Theorems 14 to 16 . If $G$ is a $H_{2}$-free graph in the class, then we can compute a branch decomposition of constant mim-width by one of Theorems 14 


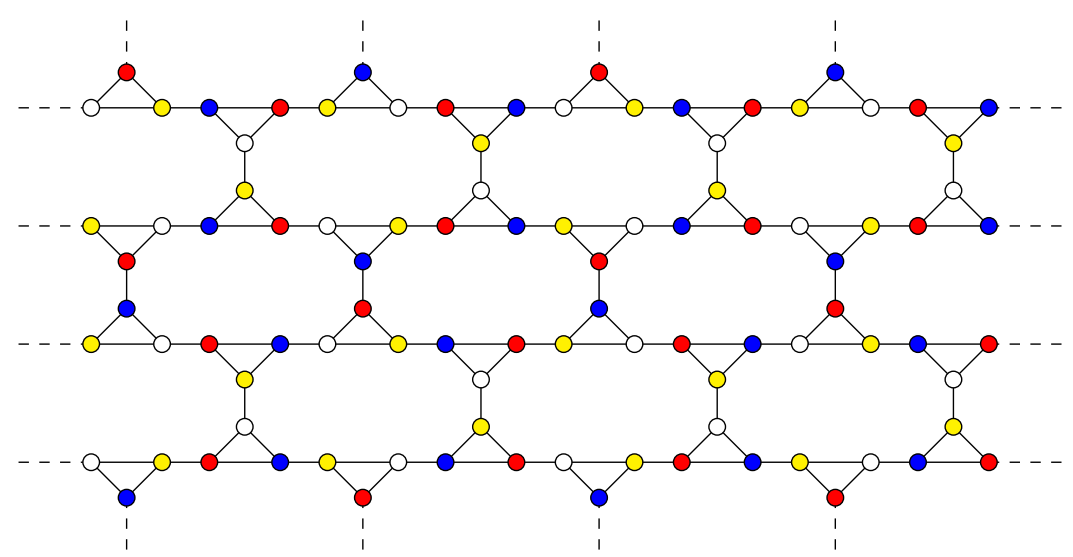

Figure 4 A particular 4-colouring of a net-wall, used in the proof of Theorem 21.

to 16 . So it remains only to show that we can compute a branch decomposition of constant mim-width for $\left(H_{1}^{\prime}, H_{2}^{\prime}\right)$-free graphs having an induced subgraph isomorphic to $H_{2}$. For example, when $H_{1}^{\prime}=2 P_{2}$ and $H_{2}^{\prime}=K_{1,3}$, we exploit the structure of $\left(2 P_{2}, K_{1,3}\right)$-free graphs having an induced $K_{3} \boxminus 3 P_{1}$ to prove the next lemma, and, together with Theorem 14, obtain Theorem 18. Similarly, when $H_{1}^{\prime}=2 P_{1}+P_{2}$ and $H_{2}^{\prime}=$ bowtie (see Figure 3 ), we use Lemma 19 and Theorem 16 to obtain Theorem 20.

- Lemma 17. Let $G$ be a connected $\left(2 P_{2}, K_{1,3}\right)$-free graph. Given $X \subseteq V(G)$ such that $G[X] \cong K_{r} \boxminus r P_{1}$ for some $r \geq 3$, where $X$ is maximal, we can construct, in $O(n)$ time, $a$ branch decomposition $(T, \delta)$ of $G$ such that $\operatorname{mimw}_{G}(T, \delta)=1$.

- Theorem 18. Let $G$ be a $\left(2 P_{2}, K_{1,3}\right)$-free graph. Then $\operatorname{mimw}(G)<6$, and one can construct, in polynomial time, a branch decomposition $(T, \delta)$ of $G$ with $\operatorname{mimw}_{G}(T, \delta)<6$.

- Lemma 19. Let $G$ be a $\left(2 P_{1}+P_{2}\right.$, bowtie)-free graph. Given $X \subseteq V(G)$ such that $G[X] \cong K_{r} \boxminus K_{r}$ for some $r \geq 5$, where $X$ is maximal, we can construct, in $O(n)$ time, a branch decomposition $(T, \delta)$ of $G$ such that $\operatorname{mimw}_{G}(T, \delta)=2$.

- Theorem 20. Let $G$ be a $\left(2 P_{1}+P_{2}\right.$, bowtie)-free graph. Then $\operatorname{mimw}(G)<R(14,3)$, and one can construct, in polynomial time, a branch decomposition $(T, \delta)$ of $G$ with $\operatorname{mimw}_{G}(T, \delta)<$ $R(14,3)$.

\section{$5 \quad$ New Unbounded Cases}

We present a number of graph classes of unbounded mim-width, starting with following two theorems (we omit the proof of the second theorem).

- Theorem 21. The class of (diamond, $5 P_{1}$ )-free graphs has unbounded mim-width.

Proof. For every integer $k$, we will construct a (diamond, $5 P_{1}$ )-free graph $G$ such that $\operatorname{mimw}(G)>k$. By Lemma 10, for any integer $k$ there exists a net-wall $W$ such that $\operatorname{mimw}(W)>4 k$. We partition the vertex set $V(W)$ into four colour classes $\left(V_{1}, V_{2}, V_{3}, V_{4}\right)$ as illustrated in Figure 4. Observe that, for each $i \in\{1,2,3,4\}$, the set $V_{i}$ is independent, and no two distinct vertices $v, v^{\prime} \in V_{i}$ have a common neighbour; that is, $N_{W}(v) \cap N_{W}\left(v^{\prime}\right)=\varnothing$.

Let $G$ be the graph obtained from $W$ by making each of $V_{1}, V_{2}, V_{3}$ and $V_{4}$ into a clique. By Lemma $4, \operatorname{mimw}(G) \geq \operatorname{mimw}(W) / 4>k$. Since any set of five vertices of $G$ contains at least two vertices in one of $V_{1}, V_{2}, V_{3}$, and $V_{4}$, and each of these four sets is a clique, $G$ is $5 P_{1}$-free. 
It remains to show that $G$ is diamond-free. First, observe that if $G[X] \cong K_{3}$ for some $X \subseteq V(G)$ with $\left|X \cap V_{i}\right| \geq 2$ for some $i \in\{1,2,3,4\}$, then, since no two vertices in $V_{i}$ have a common neighbour in $W$, it follows that $X \subseteq V_{i}$. Now, towards a contradiction, suppose $G[Y] \cong$ diamond for some $Y \subseteq V(G)$. Then $Y$ is the union of two sets $X^{\prime}$ and $X^{\prime \prime}$ that induce triangles in $G$, and $\left|X^{\prime} \cap X^{\prime \prime}\right|=2$. Since $W$ is diamond-free, we may assume that $W\left[X^{\prime}\right]$ is not a triangle. Then $X^{\prime}$ contains at least two vertices of $V_{i}$ for some $i \in\{1,2,3,4\}$. By the earlier observation, $X^{\prime} \subseteq V_{i}$. Since $\left|X^{\prime} \cap X^{\prime \prime}\right|=2$, we then have $\left|X^{\prime \prime} \cap V_{i}\right| \geq 2$, so $X^{\prime \prime} \subseteq V_{i}$, and hence $Y \subseteq V_{i}$. But this implies that $Y$ is a clique in $G$; a contradiction. So $G$ is diamond-free.

Theorem 22. The class of $\left(4 P_{1}, \overline{3 P_{1}+P_{2}}, \overline{P_{1}+2 P_{2}}\right)$-free graphs has unbounded mim-width.

Next we use the construction of a chordal bipartite graph $G^{\prime}$ from a graph $G$, given in $[11]^{2}$. Let $G=(V, E)$ be a graph. We take two copies of $V$ labelled as follows: $X=\left\{x_{v}: v \in V\right\}$ and $Y=\left\{y_{v}: v \in V\right\}$. To construct $G^{\prime}$, start with a complete bipartite graph with vertex bipartition $(X, Y)$, and add, for each edge $e \in E$ with endpoints $u$ and $v$, two paths: an $x_{u} y_{v^{-}}$ path $x_{u} q_{e} t_{e} y_{v}$, and an $x_{v} y_{u}$-path $x_{v} q_{e}^{\prime} t_{e}^{\prime} y_{u}$. For convenience, we let $Q=\bigcup_{e \in E(G)}\left\{q_{e}, q_{e}^{\prime}\right\}$ and $T=\bigcup_{e \in E(G)}\left\{t_{e}, t_{e}^{\prime}\right\}$. Observe that $(X, Y, Q, T)$ partitions $V\left(G^{\prime}\right)$; see also Figure 5 .
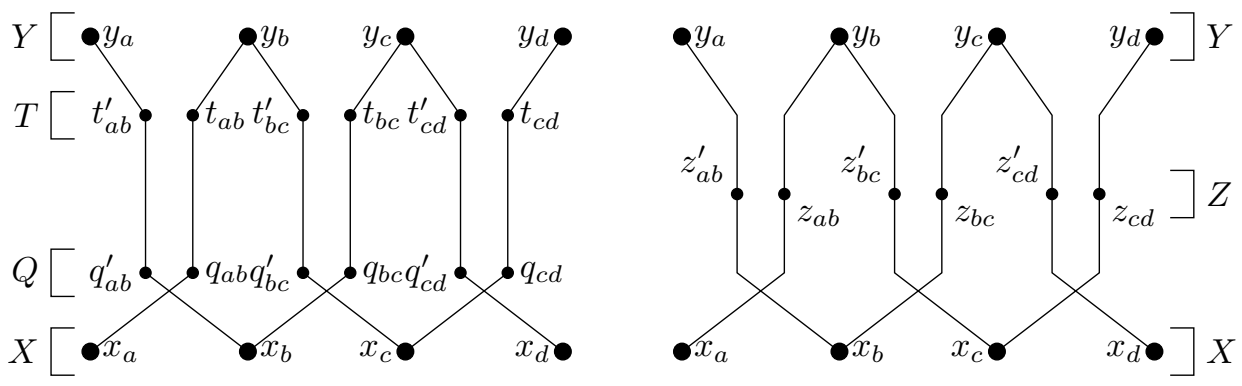

Figure 5 The graphs $G^{\prime}$ and $G^{\prime \prime}$, excluding edges between $X$ and $Y$.

We need two lemmas. The first one is due to Baron, Capelli and Mengel. We omit the proof of the second one.

- Lemma 23 ([11, Lemmas 15 and 16]). For any graph $G$, the graph $G^{\prime}$ is chordal bipartite, Moreover, if $G$ is bipartite, then $\operatorname{mimw}\left(G^{\prime}\right) \geq \operatorname{tw}(G) / 6$, where $\operatorname{tw}(G)$ denotes the treewidth of $G$.

- Lemma 24. For any graph $G$, the chordal bipartite graph $G^{\prime}$ is $\left(P_{8}, P_{3}+P_{6}, S_{1,1,5}\right)$-free.

Lemma 24 is tight in the following sense: for some graph $G$, the graph $G^{\prime}$ can contain, as an induced subgraph, $t P_{2}+P_{7}$ or $t P_{5}$ for any non-negative integer $t$, or $S_{2,2,4}$.

Theorem 25 now follows from Lemmas 23 and 24 and the fact that bipartite graphs can have arbitrarily large treewidth (see, e.g., [47]). We use Lemma 4 to obtain Theorems 26 and 27 (proofs omitted).

Theorem 25. The class of chordal bipartite $\left(P_{8}, P_{3}+P_{6}, S_{1,1,5}\right)$-free graphs has unbounded mim-width.

2 Alternatively, we could take a wall, which has bipartition classes $A$ and $B$; 2-subdivide all of its edges; and make $A$ complete to $B$. The resulting graph has the same structure as $G^{\prime}$ and can have arbitrarily large mim-width due to Theorem 8 and Lemmas 2 and 4. 

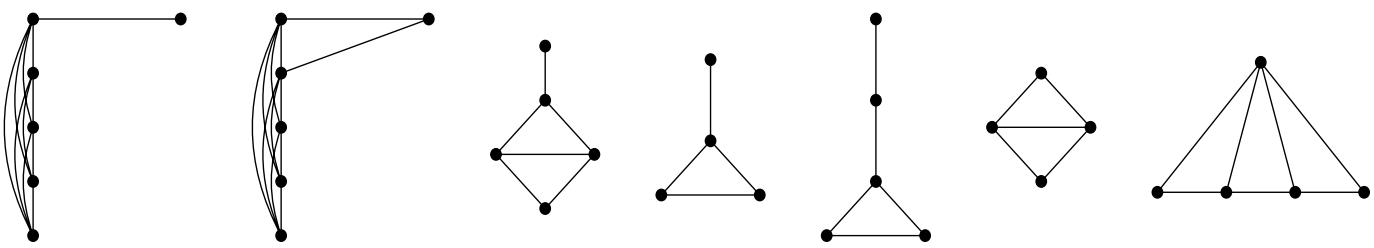

Figure 6 The graphs $K_{5} \boxminus P_{1}=\overline{K_{1,4}+P_{1}}, \overline{K_{1,3}+2 P_{1}}, \overline{S_{1,1,2}}$, paw, hammer, diamond and gem.

- Theorem 26. The class of $\left(4 P_{1}\right.$, gem, $\left.\overline{P_{1}+2 P_{2}}\right)$-free graphs has unbounded mim-width.

- Theorem 27. The class of (diamond, $2 P_{3}$ )-free graphs has unbounded mim-width.

We now describe the construction of a graph $G^{\prime \prime}$ from a graph $G=(V, E)$. This construction is similar to the construction of $G^{\prime}$; we adapt the approach taken by [11] to construct graphs with arbitrarily large mim-width. Take two copies of $V$ labelled as follows: $X=\left\{x_{v}: v \in V\right\}$ and $Y=\left\{y_{v}: v \in V\right\}$. Construct a graph $G^{\prime \prime}$ on vertex set $X \cup Y \cup Z$ where $Z=\bigcup_{e \in E(G)}\left\{z_{e}, z_{e}^{\prime}\right\}$. Start with a complete bipartite graph with vertex bipartition $(X, Y)$, and add, for each edge $e \in E$ with endpoints $u$ and $v$, two paths $x_{u} z_{e} y_{v}$ and $x_{v} z_{e}^{\prime} y_{u}$. Observe that $G^{\prime \prime}$ is 3-partite, with colour classes $(X, Y, Z)$; see also Figure 5.

The following lemma is proven by modifying the proof of Lemma 23 given in [11]. Alternatively, we could take the $n \times n$ wall $W$, which has bipartition classes $A$ and $B$; 1-subdivide each edge of $W$; and make $A$ complete to $B$. By applying Theorem 8 and Lemmas 2 and 4, we obtain a lower bound on the mim-width in terms of $n$.

- Lemma 28. If $G$ is a bipartite graph, then $\operatorname{mimw}\left(G^{\prime \prime}\right) \geq \operatorname{tw}(G) / 6$.

We use Lemma 28 to show the following theorem (proof omitted).

- Theorem 29. The class of $\left(K_{4}\right.$, diamond, $\left.P_{6}, P_{2}+P_{4}\right)$-free graphs has unbounded mim-width

\section{State of the Art}

We show the consequences of the results from Sections 3-5 for the boundedness and unboundedness of mim-width of classes of $\left(H_{1}, H_{2}\right)$-free graphs. We will also make a comparison between the results for mim-width and clique-width. In contrast to the situation where only one induced subgraph is forbidden, we note many differences when two induced subgraphs $H_{1}$ and $H_{2}$ are forbidden. Figure 6 illustrates a number of graphs used in the section.

Our first summary theorem follows from combining our results with known results from $[5,6,7,8,9,10,12,21,22,24,25,26,37,42]$ (proof details omitted). It gives all pairs $\left(H_{1}, H_{2}\right)$ for which the mim-width of the class of $\left(H_{1}, H_{2}\right)$-free graphs is bounded. This theorem gives more bounded cases than the corresponding summary theorem for boundedness of clique-width of classes of $\left(H_{1}, H_{2}\right)$-free graphs, which can be found in [23] and which we need for our proof. To get the summary theorem for clique-width, replace Cases (x)-(xv) of Theorem 30 by the more restricted case where $H_{1}=K_{s}$ and $H_{2}=t P_{1}$ for some $s, t \geq 1$.

- Theorem 30. For graphs $H_{1}$ and $H_{2}$, the mim-width of the class of $\left(H_{1}, H_{2}\right)$-free graphs is bounded and quickly computable if one of the following holds:

(i) $H_{1}$ or $H_{2} \subseteq_{i} P_{4}$,

(ii) $H_{1} \subseteq_{i}$ paw and $H_{2} \subseteq_{i} K_{1,3}+3 P_{1}, K_{1,3}+P_{2}, P_{1}+P_{2}+P_{3}, P_{1}+P_{5}, P_{1}+S_{1,1,2}$, $P_{2}+P_{4}, P_{6}, S_{1,1,3}$ or $S_{1,2,2}$, 
(iii) $H_{1} \subseteq_{i} P_{1}+P_{3}$ and $H_{2} \subseteq_{i} \overline{K_{1,3}+3 P_{1}}, \overline{K_{1,3}+P_{2}}, \overline{P_{1}+P_{2}+P_{3}}, \overline{P_{1}+P_{5}}, \overline{P_{1}+S_{1,1,2}}$, $\overline{P_{2}+P_{4}}, \overline{P_{6}}, \overline{S_{1,1,3}}$ or $\overline{S_{1,2,2}}$,

(iv) $H_{1} \subseteq_{i}$ diamond and $H_{2} \subseteq_{i} P_{1}+2 P_{2}, 3 P_{1}+P_{2}$ or $P_{2}+P_{3}$,

(v) $H_{1} \subseteq_{i} 2 P_{1}+P_{2}$ and $H_{2} \subseteq_{i} \overline{P_{1}+2 P_{2}}, \overline{3 P_{1}+P_{2}}$ or $\overline{P_{2}+P_{3}}$,

(vi) $H_{1} \subseteq_{i}$ gem and $H_{2} \subseteq_{i} P_{1}+P_{4}$ or $P_{5}$,

(vii) $H_{1} \subseteq_{i} P_{1}+P_{4}$ and $H_{2} \subseteq_{i} \overline{P_{5}}$,

(viii) $H_{1} \subseteq_{i} K_{3}+P_{1}$ and $H_{2} \subseteq_{i} K_{1,3}$,

(ix) $H_{1} \subseteq_{i} 2 P_{1}+P_{3}$ and $H_{2} \subseteq_{i} \overline{2 P_{1}+P_{3}}$,

(x) $H_{1} \subseteq_{i} 2 P_{1}+P_{2}$ and $H_{2} \subseteq_{i}$ bowtie,

(xi) $H_{1} \subseteq_{i} K_{1,3}$ and $H_{2} \subseteq_{i} 2 P_{2}$,

(xii) $H_{1} \subseteq_{i} K_{r}$ for $r \geq 1$ and $H_{2} \subseteq_{i} s P_{1}+P_{5}$ for $s \geq 0$,

(xiii) $H_{1} \subseteq_{i} K_{r} \boxminus r P_{1}$ for $r \geq 1$ and $H_{2} \subseteq_{i} 2 P_{2}$,

(xiv) $H_{1} \subseteq_{i} K_{r} \boxminus P_{1}$ for $r \geq 1$ and $H_{2} \subseteq_{i} t P_{2}$ for $t \geq 1$, or

(xv) $H_{1} \subseteq_{i} K_{r} \boxminus K_{r}$ for $r \geq 1$ and $H_{2} \subseteq_{i} s P_{1}+P_{2}$ for $s \geq 0$.

Our second summary theorem, on the unbounded cases of mim-width, follows from combining results in previous sections (proof details omitted). We let $\mathcal{S}$ be the class of graphs every connected component of which is either a subdivided claw or a path. We let $\mathcal{N}$ denote the class of graphs that contain a connected component with either a cycle of length at least 4 or at least two (not necessarily vertex-disjoint) triangles; note, for example, that $\mathcal{N}$ contains $C_{4}$, diamond, and $K_{4}$.

- Theorem 31. For graphs $H_{1}$ and $H_{2}$, the class of $\left(H_{1}, H_{2}\right)$-free graphs has unbounded mim-width if one of the following holds:

(i) $H_{1} \notin \mathcal{S}$ and $H_{2} \notin \mathcal{S}$,

(ii) $H_{1} \supseteq_{i} C_{3}$ and $H_{2} \supseteq_{i} P_{3}+P_{6}, P_{8}$ or $S_{1,1,5}$,

(iii) $H_{1} \supseteq_{i} K_{1,3}$ and $H_{2} \in \mathcal{N}$,

(iv) $H_{1} \supseteq_{i}$ diamond and $H_{2} \supseteq_{i} 5 P_{1}, P_{2}+P_{4}, 2 P_{3}$ or $P_{6}$,

(v) $H_{1} \supseteq_{i} 3 P_{1}$ and $H_{2} \supseteq_{i} 3 P_{1}, C_{5}$ or $\overline{C_{2 s+1}}$ for $s \geq 3$,

(vi) $H_{1} \supseteq_{i} 4 P_{1}$ and $H_{2} \supseteq_{i}$ gem, $\overline{3 P_{1}+P_{2}}$ or $\overline{P_{1}+2 P_{2}}$,

(vii) $H_{1} \supseteq_{i} 2 P_{2}$ and $H_{2} \supseteq_{i} C_{4}, C_{5}, K_{1,4}, 2 P_{2}, \overline{3 P_{1}+P_{2}}$ or $\operatorname{sun}_{t}$ for $t \geq 3$, or

(viii) $H_{1} \supseteq_{i} K_{4}$ and $H_{2} \supseteq_{i} P_{2}+P_{4}$ or $P_{6}$.

We note that the situation for the unbounded cases is again different from the situation for the unbounded cases of clique-width. For example, $\left(H_{1}, H_{2}\right)$-free graphs have unbounded clique-width if both $\overline{H_{1}} \notin \mathcal{S}$ and $\overline{H_{2}} \notin \mathcal{S}$ (see, for example, [26]). Take, for instance, $H_{1}=4 P_{1}$ and $H_{2}=2 P_{2}$. Then $\overline{H_{1}}=K_{4}$ and $\overline{H_{2}}=C_{4}$, and thus $\overline{H_{1}} \notin \mathcal{S}$ and $\overline{H_{2}} \notin \mathcal{S}$, so $\left(H_{1}, H_{2}\right)$ free graphs have unbounded clique-width. However, by Theorem 30-(xiii), $\left(H_{1}, H_{2}\right)$-free graphs have bounded mim-width. As $\left(\overline{H_{1}}, \overline{H_{2}}\right)$-free graphs have unbounded mim-width by Theorem 31-(i), this example also shows that the complementation operation, a standard tool for working with clique-width, cannot be used for mim-width. Consequently, for mim-width there are many more open cases than the only five open cases for clique-width [23]. In order to get a handle on the open cases for mim-width, we now present some consequences of Theorems 30 and 31.

We first note that Theorems 30 and 31 cover all pairs $\left(H_{1}, H_{2}\right)$ with $\left|V\left(H_{1}\right)\right|+\left|V\left(H_{2}\right)\right| \leq 8$.

- Corollary 32. Let $H_{1}$ and $H_{2}$ be graphs with $\left|V\left(H_{1}\right)\right|+\left|V\left(H_{2}\right)\right| \leq 8$. Then the pair $\left(H_{1}, H_{2}\right)$ satisfies Theorem 30 or Theorem 31.

We now present two open problems involving some particular open cases arising from Theorems 30 and 31. 
- Open Problem 1. Let $H_{1}$ and $H_{2}$ be forests. Then (un)boundedness of mim-width of $\left(H_{1}, H_{2}\right)$-free graphs is open if and only if

1. $H_{1}=2 P_{2}$ and $H_{2}=K_{1,3}+s P_{1}$ for $s \geq 1$, or

2. $H_{1}=2 P_{2}$ and $H_{2}=S_{1,1,2}+s P_{1}$ for $s \geq 0$.

- Open Problem 2. Let $H_{1}$ and $H_{2}$ be connected graphs. Then (un)boundedness of mimwidth of $\left(H_{1}, H_{2}\right)$-free graphs is open if and only if

1. $H_{1}=P_{5}$ and $H_{2}=\overline{S_{1,1,2}}$ or $\overline{K_{1, r}+s P_{1}}$ for $r \geq 3$ and $s \in\{1,2\}$,

2. $H_{1}=P_{7}$ or $S_{h, i, j}$ for $h \leq i \leq j \leq 4$ with $i+j \leq 6 \leq h+i+j$ and $H_{2}=C_{3}$ or paw, or

3. $H_{1}=K_{1,3}$ or $S_{1,1,2}$ and $H_{2}=$ hammer.

\section{Conclusion}

We extended the toolkit for proving (un)boundedness of mim-width of hereditary graph classes. Using the extended toolkit, we found new classes of $\left(H_{1}, H_{2}\right)$-free graphs of bounded and unbounded mim-width. We showed that the situation for mim-width of hereditary graph classes is different from the situation for clique-width, even when only two induced subgraphs $H_{1}$ and $H_{2}$ are forbidden. For future work, Open Problems 1 and 2 deserve attention. In particular, the class of $\left(P_{5}, \overline{K_{1, r}+s P_{1}}\right)$-free graphs, for $r \geq 3$ and $s \in\{1,2\}$ (Case 1 of Open Problem 2), is the only remaining infinite family. Moreover, a similar approach to Theorem 18 might be conducive to resolving further open cases where $H_{1}=2 P_{2}$.

Another interesting case is when $H_{1}=K_{r}$ for some $r$. For $r \geq 4$, Theorems 30 and 31 imply that the mim-width of the class of $\left(K_{r}, H_{2}\right)$-free graphs is bounded and quickly computable when $H_{2} \subseteq_{i} s P_{1}+P_{5}$ or $t P_{2}$, and unbounded when $H_{2} \supseteq_{i} K_{1,3}, P_{2}+P_{4}$, or $P_{6}$, or $H_{2} \notin \mathcal{S}$. It can be shown that all remaining cases belong to one infinite family: when $H_{2}=t P_{2}+u P_{3}$ for $u \geq 1$ and $t+u \geq 2$. For any $H_{2}$ such that mim-width is bounded and quickly computable for the class of $\left(K_{r}, H_{2}\right)$-free graphs, $k$-ColOuRING is polynomial-time solvable for all $k<r$ (for example, see [12] when $H_{2} \subseteq_{i} s P_{1}+P_{5}$ ). For problems having polynomial-time algorithms when mim-width is bounded and quickly computable, we obtain $n^{f(\omega(G))}$-time algorithms, for some function $f$, when restricted to $H_{2}$-free graphs; that is, XP algorithms parameterized by $\omega(G)$ (the size of the largest clique in $G$ ). Recently, Chudnovsky et al. [17] showed that for $P_{5}$-free graphs, there exists an $n^{O(\omega(G))}$-time algorithm for MAX Partial $H$-Colouring, a problem generalizing Maximum Independent Set and Odd Cycle Transversal, and which is polynomial-time solvable when mim-width is bounded and quickly computable.

One could also consider the class of $\left(r P_{1}, H_{2}\right)$-free graphs, for an integer $r$ and a graph $H_{2}$, and similarly obtain, for many problems, XP algorithms parameterized by $\alpha(G)$ for the class of $H_{2}$-free graphs, where $\alpha(G)$ is the size of the largest independent set in $G$. For $r \geq 5$, Theorems 30 and 31 imply that the mim-width of the class of $\left(r P_{1}, H_{2}\right)$-free graphs is bounded and quickly computable when $H_{2} \subseteq_{i} K_{t} \boxminus K_{t}$ for some $t$, and unbounded when $H_{2}$ is not co-bipartite, or $H_{2} \supseteq_{i}$ diamond. All unresolved cases belong to the infinite family $H_{2}=\overline{K_{s, t}+P_{1}}$ for $s, t \geq 2$ (note that if $s=t=2$, then $H_{2}=$ bowtie).

\section{References}

1 Rémy Belmonte and Martin Vatshelle. Graph classes with structured neighborhoods and algorithmic applications. Theoretical Computer Science, 511:54-65, 2013.

2 Benjamin Bergougnoux and Mamadou Moustapha Kanté. More applications of the d-neighbor equivalence: Connectivity and acyclicity constraints. Proc. ESA 2019, LIPIcs, 144:17:1-17:14, 2019 . 
3 Benjamin Bergougnoux, Charis Papadopoulos, and Jan Arne Telle. Node multiway cut and subset feedback vertex set on graphs of bounded mim-width. Proc. WG 2020, LNCS, to appear, 2020.

4 Therese C. Biedl, Erik D. Demaine, Christian A. Duncan, Rudolf Fleischer, and Stephen G. Kobourov. Tight bounds on maximal and maximum matchings. Discrete Mathematics, 285:7-15, 2004.

5 Alexandre Blanché, Konrad K. Dabrowski, Matthew Johnson, Vadim V. Lozin, Daniël Paulusma, and Viktor Zamaraev. Clique-width for graph classes closed under complementation. SIAM Journal on Discrete Mathematics, 34:1107-1147, 2020.

6 Rodica Boliac and Vadim V. Lozin. On the clique-width of graphs in hereditary classes. Proc. ISAAC 2002, LNCS, 2518:44-54, 2002.

7 Andreas Brandstädt, Tilo Klembt, and Suhail Mahfud. $P_{6}$-and triangle-free graphs revisited: structure and bounded clique-width. Discrete Mathematics $\&$ Theoretical Computer Science, 8:173-188, 2006.

8 Andreas Brandstädt, Hoàng-Oanh Le, and Raffaele Mosca. Gem-and co-gem-free graphs have bounded clique-width. International Journal of Foundations of Computer Science, 15:163-185, 2004.

9 Andreas Brandstädt, Hoàng-Oanh Le, and Raffaele Mosca. Chordal co-gem-free and ( $P_{5}$, gem)free graphs have bounded clique-width. Discrete Applied Mathematics, 145:232-241, 2005.

10 Andreas Brandstädt and Suhail Mahfud. Maximum weight stable set on graphs without claw and co-claw (and similar graph classes) can be solved in linear time. Information Processing Letters, 84:251-259, 2002.

11 Johann Brault-Baron, Florent Capelli, and Stefan Mengel. Understanding model counting for beta-acyclic CNF-formulas. Proc. STACS 2015, LIPIcs, 30:143-156, 2015.

12 Nick Brettell, Jake Horsfield, and Daniël Paulusma. Colouring $\left(s P_{1}+P_{5}\right)$-free graphs: a mim-width perspective. CoRR, abs/2004.05022, 2020. arXiv:2004. 05022.

13 Nick Brettell, Andrea Munaro, and Daniël Paulusma. List $k$-Colouring $P_{t}$-free graphs with no induced 1-subdivision of $K_{1, s}$ : a mim-width perspective. CoRR, abs/2008.01590, 2020. arXiv:2008.01590.

14 Nick Brettell, Andrea Munaro, and Daniël Paulusma. Solving problems on generalized convex graphs via mim-width. CoRR, abs/2008.09004, 2020. arXiv:2008.09004.

15 Binh-Minh Bui-Xuan, Jan Arne Telle, and Martin Vatshelle. Boolean-width of graphs. Theoretical Computer Science, 412:5187-5204, 2011.

16 Binh-Minh Bui-Xuan, Jan Arne Telle, and Martin Vatshelle. Fast dynamic programming for locally checkable vertex subset and vertex partitioning problems. Theoretical Computer Science, 511:66-76, 2013.

17 Maria Chudnovsky, Jason King, Michal Pilipczuk, Pawel Rzazewski, and Sophie Spirkl. Finding large $H$-colorable subgraphs in hereditary graph classes. Proc. ESA 2020, LIPIcs, 173:35:1-35:17, 2020.

18 Maria Chudnovsky, Sophie Spirkl, and Mingxian Zhong. List-3-coloring $P_{t}$-free graphs with no induced 1-subdivision of $K_{1, s}$. Discrete Mathematics, 343:112086, 2020.

19 Bruno Courcelle and Stephan Olariu. Upper bounds to the clique width of graphs. Discrete Applied Mathematics, 101:77-114, 2000.

20 Jean-François Couturier, Petr A. Golovach, Dieter Kratsch, and Daniël Paulusma. List coloring in the absence of a linear forest. Algorithmica, 71:21-35, 2015.

21 Konrad K. Dabrowski, François Dross, and Daniël Paulusma. Colouring diamond-free graphs. Journal of Computer and System Sciences, 89:410-431, 2017.

22 Konrad K. Dabrowski, Shenwei Huang, and Daniël Paulusma. Bounding clique-width via perfect graphs. Journal of Computer and System Sciences, 104:202-215, 2019.

23 Konrad K. Dabrowski, Matthew Johnson, and Daniël Paulusma. Clique-width for hereditary graph classes. London Mathematical Society Lecture Note Series, 456:1-56, 2019. 
24 Konrad K. Dabrowski, Vadim V. Lozin, and Daniël Paulusma. Clique-width and well-quasiordering of triangle-free graph classes. Joournal of Computer and System Sciences, 108:64-91, 2020.

25 Konrad K. Dabrowski, Vadim V. Lozin, Rajiv Raman, and Bernard Ries. Colouring vertices of triangle-free graphs without forests. Discrete Mathematics, 312:1372-1385, 2012.

26 Konrad K. Dabrowski and Daniël Paulusma. Clique-width of graph classes defined by two forbidden induced subgraphs. The Computer Journal, 59:650-666, 2016.

27 Fedor V. Fomin, Petr A. Golovach, and Jean-Florent Raymond. On the tractability of optimization problems on H-graphs. Proc. ESA 2018, LIPIcs, 30:1-14, 2018.

28 Esther Galby and Andrea Munaro. Approximating Independent Set and Dominating Set on VPG graphs. CoRR, abs/2004.07566, 2020. arXiv:2004.07566.

29 Esther Galby, Andrea Munaro, and Bernard Ries. Semitotal domination: New hardness results and a polynomial-time algorithm for graphs of bounded mim-width. Theoretical Computer Science, 814:28-48, 2020.

30 Georg Gottlob, Petr Hliněný, Sang-il Oum, and Detlef Seese. Width parameters beyond tree-width and their applications. The Computer Journal, 51:326-362, 2008.

31 Frank Gurski. The behavior of clique-width under graph operations and graph transformations. Theory of Computing Systems, 60:346-376, 2017.

32 Venkatesan Guruswami and C. Pandu Rangan. Algorithmic aspects of clique-transversal and clique-independent sets. Discrete Applied Mathematics, 100:183-202, 2000.

33 Lars Jaffke, O-joung Kwon, Torstein J. F. Strømme, and Jan Arne Telle. Mim-width III. Graph powers and generalized distance domination problems. Theoretical Computer Science, 796:216-236, 2019.

34 Lars Jaffke, O-joung Kwon, and Jan Arne Telle. Mim-width I. Induced path problems. Discrete Applied Mathematics, 278:153-168, 2020.

35 Lars Jaffke, O-joung Kwon, and Jan Arne Telle. Mim-width II. The Feedback Vertex Set problem. Algorithmica, 82:118-145, 2020.

36 Öjvind Johansson. Clique-decomposition, NLC-decomposition, and modular decomposition relationships and results for random graphs. Congressus Numerantium, 132:39-60, 1998.

37 Marcin Kamiński, Vadim V. Lozin, and Martin Milanič. Recent developments on graphs of bounded clique-width. Discrete Applied Mathematics, 157:2747-2761, 2009.

38 Dong Yeap Kang, O-joung Kwon, Torstein J. F. Strømme, and Jan Arne Telle. A width parameter useful for chordal and co-comparability graphs. Theoretical Computer Science, 704:1-17, 2017.

39 Iyad Kanj, Michael J. Pelsmajer, Marcus Schaefer, and Ge Xia. On the induced matching problem. Journal of Computer and System Sciences, 77(6):1058-1070, 2011.

40 Vadim V. Lozin and Dieter Rautenbach. On the band-, tree-, and clique-width of graphs with bounded vertex degree. SIAM Journal on Discrete Mathematics, 18:195-206, 2004.

41 Stefan Mengel. Lower bounds on the mim-width of some graph classes. Discrete Applied Mathematics, 248:28-32, 2018.

42 Sang-il Oum and Paul D. Seymour. Approximating clique-width and branch-width. Journal of Combinatorial Theory, Series B, 96:514-528, 2006.

43 Andrzej Proskurowski and Jan Arne Telle. Algorithms for vertex partitioning problems on partial $k$-trees. SIAM Journal on Discrete Mathematics, 10:529-550, 1997.

44 Michaël Rao. Clique-width of graphs defined by one-vertex extensions. Discrete Mathematics, 308:6157-6165, 2008.

45 Neil Robertson and Paul D. Seymour. Graph minors. X. Obstructions to tree-decomposition. Journal of Combinatorial Theory, Series B, 52:153-190, 1991.

46 Sigve Hortemo Sæther and Martin Vatshelle. Hardness of computing width parameters based on branch decompositions over the vertex set. Theoretical Computer Science, 615:120-125, 2016.

47 Martin Vatshelle. New Width Parameters of Graphs. PhD thesis, University of Bergen, 2012. 